\title{
IMPACTO DEL EJERCICIO FÍSICO EN EL MANEJO DE LA DISLIPIDEMIA DIABÉTICA
}

\author{
IMPACT OF PHYSICAL EXERCISE IN THE \\ MANAGEMENT OF DIABETIC DISLIPIDEMIA
}

Martín Rodríguez

\begin{abstract}
RESUMEN
La actividad física genera una mejor aptitud cardiorrespiratoria o fitness cardiorrespiratorio (FCR). Un mayor FCR se asocia a numerosos beneficios: menor riesgo de mortalidad cardiovascular y por todas las causas, menor riesgo de hipertensión arterial (HTA) y de diabetes mellitus tipo 2 (DM2), menor riesgo de diversos cánceres y un menor riesgo de perfil lipídico desfavorable. En este último punto, la dificultad de realizar estudios clínicos aleatorizados de magnitud suficiente hace que la evidencia sea moderada. No obstante, puede afirmarse que los efectos del ejercicio sobre el perfil lipídico en pacientes DM2 son positivos, con un beneficio más manifiesto de los ejercicios aeróbicos y combinados, y sobre todo cuando el volumen y la intensidad del ejercicio son mayores.
\end{abstract}

Palabras clave: diabetes mellitus tipo 2; ejercicio; actividad física; dislipidemia diabética.

Revista de la Sociedad Argentina de Diabetes 2018; Vol. 52 (86-93)

\begin{abstract}
Physical activity generates a better cardiorespiratory fitness (CRF). A greater CRF is associated with numerous benefits: lower risk of cardiovascular mortality and all causes, lower risk of hypertension, type 2 diabetes, lower risk of various cancers and a lower risk of unfavorable lipid profile. In this last point, the difficulty of conducting randomized clinical trials of enough magnitude makes the evidence moderate. However, it can be affirmed that the effects of exercise on the lipid profile in type 2 diabetes patients are positive, with a more evident benefit of aerobic and combined exercises and especially when the volume and intensity of exercise are greater.
\end{abstract}

Key words: type 2 diabetes, exercise, physical activity, diabetic dyslipidemia.

Revista de la Sociedad Argentina de Diabetes 2018; Vol. 52 (86-93)
Médico especializado en Medicina Interna y Diabetes, Profesor Titular de Endocrinología, Metabolismo y Nutrición, Facultad de Ciencias Médicas, Universidad Nacional de Cuyo, Mendoza, Argentina

Contacto del autor: Martín Rodríguez

E-mail: clindiab@gmail.com
Correspondencia: Boulogne Sur Mer 1585 (CP 5500), Mendoza, Argentina

Fecha de trabajo recibido: 11/01/19

Fecha de trabajo aceptado: 04/02/19

Conflictos de interés: el autor declara que no existe conflicto de interés

\section{INTRODUCCIÓN}

Los cuatro pilares de la terapia para la diabetes mellitus tipo 2 (DM2) consisten en: educación, medicación, intervención nutricional y actividad física. El ejercicio regular se recomienda fuertemente en todas las guías de práctica clínica en diabetes, sin embargo, existe un gran debate sobre cómo lograr la adherencia, los alcances del beneficio, el volumen de ejercicio necesario, y la intensidad y el tipo de ejercicio que beneficiará más a esta población vulnerable. Debido a la complejidad y el gasto que implican los ensayos clínicos, gran parte de la información proviene de estudios de observación, y los escasos estudios controlados y aleatorizados tienen poca influencia por el pequeño tamaño de las muestras y la heterogeneidad en el diseño.

\section{Ejercicio, fitness cardiorrespiratorio y mortalidad cardiovascular}

El fitness cardiorrespiratorio (FCR) o aptitud cardiorrespiratoria es la capacidad de los sistemas circulatorio y respiratorio para suministrar oxígeno a los músculos esqueléticos durante la actividad 
física sostenida. La medida primaria de FCR es el consumo máximo de $\mathrm{O} 2$ ( $\mathrm{VO} 2 \mathrm{max}$ ) expresado en $\mathrm{mL}$ de $\mathrm{O} 2$ por $\mathrm{kg}$ de peso corporal y por minuto o en equivalentes metabólicos METs, donde 1 MET equivale a $3,5 \mathrm{mLO} 2 / \mathrm{kg} / \mathrm{min}$ (1 MET representa el consumo de $\mathrm{O} 2$ durante el reposo completo). Existe una creciente evidencia de que un bajo FCR es un fuerte predictor de mortalidad en igual o mayor medida que otros factores de riesgo establecidos, como el tabaco, la hipertensión, el colesterol de LDL elevado y la DM2. Debería agregarse el FCR a los factores de riesgo tradicionales para mejorar la validez de la predicción de riesgo. En 2016, la American Heart Association estableció que el FCR debe considerarse como un signo vital y que debe evaluarse de forma rutinaria como parte de la práctica clínica'.

EI FCR refleja la capacidad integrada para transportar oxígeno desde la atmósfera hasta las mitocondrias. Por lo tanto, cuantifica la capacidad funcional de un individuo y depende de una cadena unida de procesos que incluyen: ventilación y difusión pulmonar, función ventricular derecha e izquierda, acoplamiento ventricular-arterial, capacidad vascular para acomodar y transportar la sangre del corazón y coincidir con precisión con los requisitos de oxígeno y la capacidad de las células musculares para recibir y utilizar el oxígeno y los nutrientes suministrados por la sangre, así como para comunicar estas demandas metabólicas al centro del control cardiorrespiratorio.

En este sentido el FCR se relaciona directamente con la función integrada de numerosos sistemas $y$, por lo tanto, se considera un reflejo de la salud corporal total. Aproximadamente la mitad del FCR se considera atribuible a factores hereditarios ${ }^{2}$; de manera similar, la contribución de la herencia a la respuesta del FCR a la actividad física se aproxima al 50\%. El FCR puede establecerse directamente al medir los gases inspirados y expirados durante un esfuerzo máximo, o estimarse a partir de pruebas de esfuerzo sin medición de gases. Se puede determinar, también, con mediana exactitud, sin hacer pruebas de esfuerzo, a partir de calculadores que incluyen variables clínicas y el grado de actividad física regular (género, peso, talla, circunferencia de cintura, frecuencia cardíaca -FC- en reposo, FC máxima calculada, frecuencia e intensidad de actividad física regular). Uno de ellos, el World Fitness Level de la Universidad Tecnológica de Noruega, puede emplearse fácilmente online (https://www.worldfitnesslevel.org/\#/).

Un FCR $<5$ METs se asocia con alta mortalidad, niveles >a 10 METs se asocian con mayor sobrevida. Llegar a un VO2max mayor a 13 METs determina un excelente pronóstico independientemente de la condición patológica que se tenga (por ejemplo, diabetes). A 18-20 METs sólo llegan los atletas de élite en deportes de fondo ${ }^{3}$. Leves incrementos del FCR (1-2 METs) se asocian con considerable reducción de la mortalidad cardiovascular (10 a 30\%).

En un estudio 4 realizado por Blair et al. en 1.263 varones DM2 seguidos por 15 años, cada MET de aumento del FCR se asoció con un 25\% de menor riesgo de mortalidad por todas las causas en un análisis multivariado $(p<0,001)$. Las recientes guías publicadas online en la revista JAMA "The Physical Activity Guidelines for Americans"s reafirman que un mejor FCR se asocia a numerosos beneficios: menor riesgo de mortalidad por todas las causas, menor riesgo de mortalidad cardiovascular, menor riesgo de enfermedad cerebrovascular, menor riesgo de HTA y DM2, menor riesgo de perfil lipídico desfavorable, menor riesgo de cáncer de vejiga, mama, colon, endometrio, esófago, riñón, pulmón y estómago, mejora cognitiva con reducción del riesgo de demencia, incluyendo la enfermedad de Alzheimer, mejoría en la calidad de vida, reducción de la ansiedad y del riesgo de depresión, mejora del sueño, mayor pérdida de peso cuando se asocia a dieta, prevención de la reganancia de peso, mejora de la salud ósea, mejora de la disfunción física, menor riesgo de caídas y menor riesgo de injuria por caídas en adultos mayores.

Las observaciones soportan la noción de que una deteriorada regulación de las vías oxidativas en las mitocondrias tal vez sea el factor común que una a un reducido FCR con un mayor riesgo cardiovascular y metabólico.

Aunque se comentó anteriormente que el FCR y la respuesta del FCR al ejercicio tienen un fuerte componente hereditario, una revisión Cochrane de 2005 demostró claramente que el aumento de la actividad física es efectivo para incrementar el FCR $^{6}$. Los esfuerzos para mejorar el FCR deben convertirse en una parte esencial de la práctica clínica (por ejemplo, ser un "signo vital" aceptado) y de la formación médica continua, incluso en la formación de grado en las escuelas de Medicina.

La intensidad y/o el volumen de ejercicio se expresan en METs y/o en calorías. Si una activi- 
dad tiene 5 METs significa que el consumo de $\mathrm{O} 2$ es cinco veces superior al consumo de $\mathrm{O} 2$ que al estar en reposo $(5 \times 3,5=17,5 \mathrm{~mL} / \mathrm{kg} / \mathrm{min}$ de $\mathrm{O} 2)$. En intensidad, por ejemplo, ciclismo moderado 7,5 METs, caminar en forma vigorosa 5-6 METs, caminar a moderada intensidad 3-4 METs, correr 8 a 10 METs, labores de jardinería 3-4 METs, pilates o Tai Chi 3 METs, ejercicios de fuerza 5 METs.

En la cuantificación del volumen de actividad física se utilizan medidas como METs-min-semana o METs-h-semana o calorías. Por ejemplo, si una persona camina a moderada intensidad 30 minutos (min), cinco veces por semana (150 min), caminar a moderada intensidad son 4 METs multiplicado por 150 min, significa que hace 600 METs-min-sem. Seiscientos METs-min-sem es el corte que emplea la International Physical Activity Questionnaire (IPAQ) para definir, por debajo del mismo, a la baja actividad física. Este corte de 600 METs-min-sem se ha usado en numerosos estudios epidemiológicos. Por ejemplo, Argentina lo empleó en la Encuesta Nacional de Factores de Riesgo de 2013 y por ella se definió que el 54,7\% de la población mayor de 18 años tiene un bajo nivel de actividad física.

$\mathrm{Si}$ tomamos otro ejemplo una persona muy activa de $80 \mathrm{~kg}$ que camina en forma rápida 60 min tres veces por semana (60 $\mathrm{min} \times 3$ veces $\times 6$ METs=1.080 METs-min-sem), va a pilates una hora dos veces a la semana (60 min $\times 2$ veces $\times 3$ METs = 360 METs-min-sem) y hace $2 \mathrm{~h}$ de jardinería por semana $(60$ min $\times 2$ veces $\times 4$ METs $=480$ METs-minsem) realiza en total 1.920 METs-min-sem, lo cual para IPAQ es actividad física moderada (600 a 3.000 METs-min-sem). Alta intensidad para IPAO es más de 1.500 METs-min-sem de sólo ejercicios vigorosos o más de 3.000 METs-min-sem de combinación de ejercicios moderados y vigorosos. Si dividimos 1.920 METs-min-sem por 60, serán 32 METs-h-sem, que es otra forma de verlo expresado en la literatura.

Otro modo de cuantificar el volumen de actividad es el grado de calorías quemadas. Para ello se emplea la fórmula METs $\times 3,5 \times$ peso en $\mathrm{kg} \div 200=$ calorías quemadas. Por ejemplo, esta persona que hizo 1.920 METs-min-sem $(1.920$ × 3,5 × $80 \div 200)$ quemó $2.688 \mathrm{kcal}$ al realizar ejercicios durante la semana.

\section{Ejercicio y dislipidemia diabética}

Probablemente uno de los beneficios del ejercicio en reducir el riesgo cardiovascular al mejorar el FCR o por otros mecanismos, sea a través del impacto en los componentes de la dislipidemia diabética.
La alta producción hepática de lipoproteínas de muy baja densidad (VLDL) es uno de los principales mecanismos de la dislipidemia diabética. El tejido adiposo libera ácidos grasos libres no esterificados (AGNE) a la sangre a través de la lipólisis. En presencia de insulinorresistencia se produce una elevada cantidad de AGNE en ayunas y postprandial resultado de una inhibición reducida de dicha lipólisis y la consecuente llegada de gran cantidad de AGNE al hígado.

La grasa originada de la ingesta es transportada por quilomicrones. El tejido adiposo y el músculo esquelético captan ácidos grasos de esos quilomicrones por acción de la lipoproteína-lipasa (LPL), y los remanentes de quilomicrones luego son captados por el hígado. Sin embargo, cuando la disponibilidad de grasa dietaria es muy alta, la liberación de AGNE excede la capacidad de captación del tejido adiposo y del músculo, y son tomados por el hígado. Por otro lado, la hiperinsulinemia incrementa la lipogénesis de novo (LDN) por mayor acción del elemento regulador de esterol que se une a la proteína-1 (EREUP-1, SREBP-1 su sigla en inglés), ácido graso sintasa (AGS, FAS su sigla en inglés) y acetil-CoA carboxilasa (ACC). Para compensar el aumento de grasa hepática captada y sintetizada se incrementa la secreción de triglicéridos (TAG) vía síntesis de VLDL y la oxidación mitocondrial. Existen evidencias de que el entrenamiento físico disminuye los AGNE en ayunas y postprandial, lo más probablemente vía descenso de la lipólisis tisular.

Además el ejercicio crónico incrementa la captación de AGNE por el músculo esquelético y como consecuencia disminuye la disponibilidad hepática de AGNE. Una mayor actividad de la LPL en el músculo esquelético con el entrenamiento físico aumenta la captación de los AGNE provenientes de quilomicrones, lo cual también reduce el flujo graso al hígado.

En humanos el ejercicio crónico reduce los niveles de insulinemia, sugiriendo que el entrenamiento físico, por este efecto, podría descender la actividad de LDN, lo cual es sustentado por estudios en animales que demuestran un descenso de la actividad de AGS y ACC. Incluso los datos en animales demuestran que, con el entrenamiento, se produce un descenso del contenido de lípidos intrahepáticos y esto ocurre en presencia de un aumento del coactivador $\gamma$ del receptor activado por el proliferador de peroxisoma 1- $\alpha$ (PGC-1 $\alpha$ ) y un aumento del contenido de proteínas mitocondriales citocromo $\mathrm{c}$ 
(Cit c), $\beta$-hidroxiacil-CoA deshidrogenasa ( $\beta$-HAD) y citrato sintasa (CS), empleadas como marcadores de función mitocondrial. El entrenamiento también desciende la secreción de TAG-VLDL posiblemente como consecuencia de una menor acumulación de TAG intrahepáticos (Figura 1).

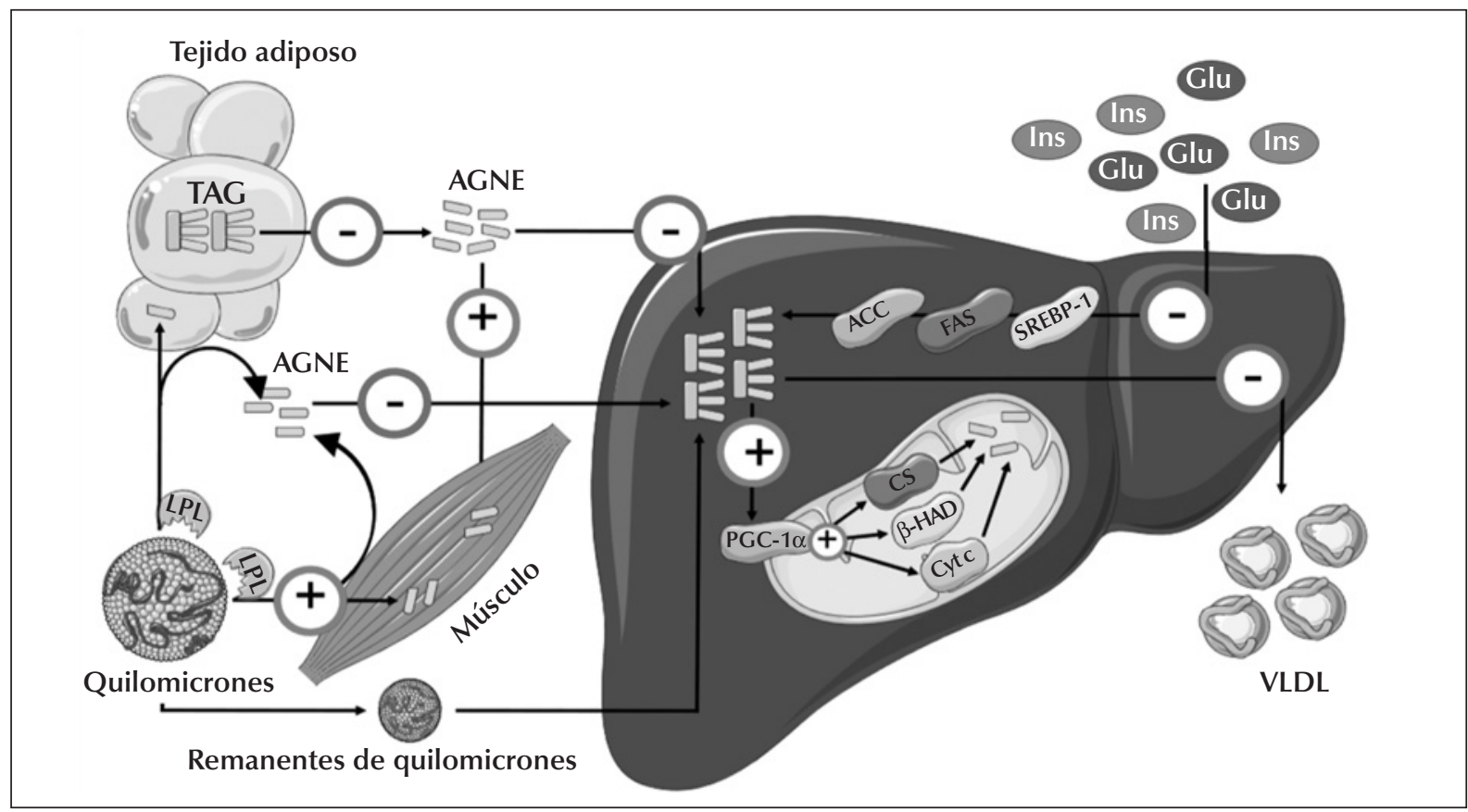

$\odot$

Acciones reducidas por el ejercicio.

Acciones aumentadas por el ejercicio.

TAG: triglicéridos; AGNE: ácidos grasos libres no esterificados; ACC: acetil-CoA carboxilasa; FAS: ácido graso sintasa (AGS); SREBP-1: elemento regulador de esterol que se une a proteína-1 (EREUP-1); PGC-1 $\alpha$ : proliferador de peroxisoma 1- $\alpha$; CS: citrato sintasa; $\beta$-HAD: $\beta$-hidroxiacil-CoA deshidrogenasa; Cyt c: citocromo C; VLDL: lipoproteínas de muy baja densidad; Glu: glucosa; Ins: insulina.

Figura 1: Vías involucradas en el metabolismo de los lípidos hepáticos y el efecto del entrenamiento físico.

El impacto de las intervenciones de ejercicios aeróbicos y de resistencia en el perfil lipídico de las personas con DM2 ha sido difícil de determinar debido a numerosos estudios a veces pequeños y conflictivos. Por ejemplo el Italian Diabetes Exercise Study (IDES) de Balducci et al. ${ }^{8}(\mathrm{n}=606)$, citado como referencia principal sobre el efecto del ejercicio sobre lípidos en las Guías Canadienses de Diabetes, demostró que el ejercicio combinado aeróbico y de resistencia con un volumen de 12,4 MET-h-sem ( $\approx 750$ MET-min-sem y $\approx 1.000 \mathrm{kcal} / \mathrm{sem}$ ) expresó una elevación significativa del HDL-C de $+3,7 \mathrm{mg} / \mathrm{dL}$ $(p<0,001)$ y un descenso de LDL-C de $-9,6 \mathrm{mg} / \mathrm{dL}$ $(p=0,003)$ comparado con un grupo control. Sin embargo, diferentes metaanálisis y revisiones sistemáticas, que incluyeron al IDES, no arribaron a las mismas conclusiones.
Se han realizado cuatro metaanálisis sobre el impacto del ejercicio en el perfil lipídico sobre población DM2. En uno de ellos, Chudyc y Petrella evaIuaron 34 estudios que cumplieron con los criterios de inclusión: DM2 adultos adjudicados en forma aleatoria a programas de ejercicios aeróbicos y/o de resistencia con un mínimo de $1.000 \mathrm{kcal}$ quemadas por semana ( $\approx$ 700-800 METs-min-sem), y comparados con un grupo control sin prescripción de ejercicio. De los 34 artículos la mayoría incluyó ejercicios sólo aeróbicos, otros sólo de resistencia u otros ejercicios combinados. También había gran variabilidad en la frecuencia prescripta (una a siete veces por semana), duración de las sesiones (40 a $75 \mathrm{~min}$ ), intensidad (entre 50 y $85 \%$ de la VO2Max) y duración de los programas (dos meses a un año). Este metaanálisis encontró 
que el entrenamiento aeróbico y el combinado se relacionan con mejoras estadísticamente significativas en el control glucémico $(-0,6 \%$ y $-0,67 \%$ de $\mathrm{HbA} 1 \mathrm{c}$, respectivamente) y los ejercicios de resistencia solos $-0,33 \%$ (NS). La presión arterial sistólica (PAS) se redujo en forma significativa con ejercicios aeróbicos y combinados $(-6 \mathrm{mmHg}$ y $-3,59 \mathrm{mmHg}$ respectivamente) sin efectos significativos de los ejercicios de resistencia solos. También la circunferencia de cintura se redujo en forma significativa con los ejercicios aeróbicos y los combinados $(-3,1 \mathrm{~cm})^{9}$. En cuanto a los lípidos, el ejercicio aeróbico solo o combinado con ejercicios de resistencia no se asoció en forma significativa a cambios en el HDL-C ni en LDL-C, sin embargo, hubo un descenso significativo de $27 \mathrm{mg} / \mathrm{dL}$ de triglicéridos. Otros estudios en individuos no DM2, con sobrepeso/obesidad y moderada dislipidemia, detectaron mejor impacto en lípidos, y los autores de este metaanálisis señalan que es probable que se necesiten mayores niveles de actividad física para afectar más positivamente los niveles de lípidos en personas con DM2.

Con un enfoque similar, Hayashino et al. ${ }^{10}$ realizaron un metaanálisis de estudios randomizados y controlados para examinar los efectos del ejercicio supervisado en el perfil de lípidos y en la presión arterial en población DM2. De Medline, Embase y del Registro de Estudios Controlados Cochrane determinaron aquellos con intervención con ejercicio (aeróbico, de resistencia o combinado) supervisado por al menos durante ocho semanas con definición del tipo, intensidad, frecuencia y duración en adultos DM2 mayores de 18 años. El diseño fue sumamente minucioso en cuanto a la extracción de datos, análisis de covariables (edad, sexo, antigüedad de la diabetes, IMC, duración de las sesiones y del programa, etc.), calidad de las determinaciones y ponderación por el tamaño de las muestras. De 2.190 potenciales estudios identificaron 42 que reunían los criterios de inclusión, con 58 ramas de tratamiento (32 aeróbico, 12 resistencia y 14 combinado). En total fueron 2.808 pacientes con una edad promedio de 56,7 años, $51 \%$ mujeres y una duración promedio de la diabetes de 6,1 años. El volumen de ejercicio promedio fue de 13,5 METs-h-sem ( $\approx 800$ METs-min-sem o $\approx 1.000 \mathrm{Kcal}$-sem). En forma global hubo aumento de HDL-C de $+1,54 \mathrm{mg} / \mathrm{dL}(\mathrm{p}<0,001)$ y por tipo de ejercicio sólo significativo en los estudios con ejercicios combinados $(+3,1 \mathrm{mg} / \mathrm{dL} p=0,008)$. La acti- vidad física fue más efectiva en elevar HDL-C en los mayores de 60 años (+3,9 mg/dL) que en los menores de 60 años (+0,39 mg/dL). El ejercicio en forma global redujo LDL-C -6,2 mg/dL sin diferencias significativas entre las diferentes formas de ejercicio. Los programas con más de 40 semanas de duración redujeron LDL-C más que los programas más breves $(-13,14 \mathrm{mg} / \mathrm{dL}$ vs $-1,2 \mathrm{mg} / \mathrm{dL})$. A diferencia de la revisión sistemática de Chudyc y Petrella ya comentada, en este metaanálisis no se encontró asociación entre el ejercicio estructurado y el descenso de triglicéridos $(-2,65 \mathrm{mg} / \mathrm{dL}$ NS). En lo que sí hubo coincidencia fue en el descenso de la PAS y en HbA1c $(-2,42 \mathrm{mmHg}$ y $-0,51 \%$ respectivamente). Este metaanálisis concluye que el ejercicio estructurado, sin intervención nutricional, fue efectivo en mejorar la presión arterial, descender LDL-C y aumentar HDL-C en personas con DM2. Los programas de mayor extensión con sesiones más prolongadas se asociaron a mejores resultados.

El ejercicio aeróbico ha sido la modalidad más estudiada. El mismo utiliza grandes grupos musculares e incluye: caminata, trote, ciclismo y natación, entre otros. Sin embargo el $80 \%$ de las personas con DM2 tiene sobrepeso u obesidad, y muchos presentan problemas de movilidad, neuropatía periférica o problemas visuales, y por lo tanto no logran el volumen e intensidad requeridos en los ejercicios aeróbicos. Los ejercicios de resistencia usan la fuerza muscular de músculos localizados en un tiempo breve y han recibido mayor atención en la última década. Existe creciente evidencia que soporta su beneficio en personas con DM2 y de hecho ya están incorporadas en varias de las guías de práctica clínica como la canadiense. Esta guía resalta varios beneficios de los ejercicios de resistencia: mejoría en el control glucémico, disminución de la insulinorresistencia, aumento de la fuerza muscular, de la masa magra muscular y de la densidad mineral ósea, lo que permite un mejor estado funcional y prevención de la sarcopenia y osteoporosis ${ }^{11}$; $y$ en sus recomendaciones establecen: las personas con diabetes (incluyendo adultos mayores) deberían realizar ejercicios de resistencia preferiblemente tres veces por semana junto con ejercicios aeróbicos acompañados de una inicial instrucción y una periódica supervisión.

Sobre todo en adultos mayores se considera más relevante, al evaluar la calidad y expectativa de vida, el índice de masa muscular (IMM=Kg de músculo 
corporal/talla $\left.{ }^{2}\right)$ que el índice de masa corporal (IMC) ${ }^{12}$. La European Working Group on Sarcopenia in Older People considera sarcopenia, en varones adultos mayores, un $\mathrm{IMM}<8,87 \mathrm{~kg} / \mathrm{m}^{2}$, una velocidad de marcha $<0,8 \mathrm{~m} / \mathrm{seg}$ y una fuerza de hand grip $<30 \mathrm{~kg}$, y en mujeres un IMM $<6,42 \mathrm{~kg} / \mathrm{m}^{2}$, una velocidad de marcha $<0,8 \mathrm{~m} / \mathrm{seg}$ y una fuerza de hand grip $<20 \mathrm{~kg}^{13}$.

Además de los metaanálisis ya referidos, una revisión sistemática de Yang et al., publicada en 2014 ${ }^{14}$, hizo mayor hincapié en las diferencias metabólicas, incluyendo lípidos, comparando estas dos modalidades de ejercicio entre sí. Con este criterio detectaron 12 estudios con 626 pacientes DM2, de diferentes países con gran variedad en edad, duración de la diabetes, grado de control glucémico inicial, período de la intervención, frecuencia, intensidad y duración de las sesiones. HbA1c mostró disminución en ambos tipos de ejercicios $(-0,32 \%$ en los de resistencia y $-0,42 \%$ en los aeróbicos) pero con muy marcada heterogeneidad. En lípidos hubo datos de 422 pacientes sobre LDL-C, HDL-C y triglicéridos. En los pacientes del grupo de ejercicio de resistencia hubo entre el comienzo y el fin del programa una disminución del LDL-C de $-1,9 \mathrm{mg} / \mathrm{dL}$ y en el grupo de ejercicio aeróbico fue de $-3,09 \mathrm{mg} / \mathrm{dL}$. Respecto de las modificaciones en HDL-C, los cambios en los grupos de resistencia y aeróbico fueron $+0,77 \mathrm{mg} / \mathrm{dL}$ y $+1,16 \mathrm{mg} / \mathrm{dL}$ respectivamente. En triglicéridos se produjo una disminución en los ejercicios de resistencia de
$-29,2 \mathrm{mg} / \mathrm{dL}$ y en los aeróbicos $-12,4 \mathrm{mg} / \mathrm{dL}$. En todos los casos con gran heterogeneidad y sin diferencias clínicamente significativas.

El cuarto metaanálisis, que evaluó lípidos y otros factores de riesgo, es el de Schwingshackl et al. ${ }^{15}$. En este análisis se compararon las diferencias entre aeróbicos solos (AS) vs resistencia solos (RS), combinados ( $A+R)$ vs aeróbicos solos (AS), y combinados $(A+R)$ vs resistencia solos (RS). Lo diferencial de este metaanálisis fue que no hubo comparación con un grupo control sedentario y que sólo se incorporaron estudios con ejercicios supervisados, además de ser aleatorizados y con un mínimo de intervención de ocho semanas. Sólo 14 ( $n=915)$ cumplieron los estrictos criterios de inclusión. Los AS fueron más efectivos que los RS en bajar $\mathrm{HbA} 1 \mathrm{c}$ (diferencia de -0,2\% $p=0,0007$ ), pero los $A+R$ fueron más efectivos aún que los AS (diferencia de $-0,17 p=0,02$ ) y que los RS (diferencia de $-0,62 \% p=0,0002$ ). No hubo diferencia significativa entre las modificaciones en lípidos generadas por AS y RS. La única diferencia significativa fue mayor reducción de triglicéridos entre $A+R$ vs $R S$ (diferencia de $-25 \mathrm{mg} / \mathrm{dL}$ $p=0,003)$. Los autores concluyen que los resultados sugieren que los ejercicios combinados $A+R$ podrían ser más eficaces en mejorar el control glucémico y los lípidos, pero que la interpretación respecto de la relevancia clínica es limitada dada la baja calidad de los estudios.

\begin{tabular}{|c|c|c|c|c|c|c|c|}
\hline Estudio & $\mathbf{N}$ & Intervención & A1c & CT & LDL-C & HDL-C & TGA \\
\hline $\begin{array}{l}\text { Balducci } \\
\text { et al. }{ }^{7}\end{array}$ & 606 pacientes & $\begin{array}{l}\approx 750 \text { MET-min-sem C } \\
\text { vs no ejercicio }\end{array}$ & $-0,3 \%$ & $-5,3 \mathrm{mg} / \mathrm{dL}$ & $-9,6 \mathrm{mg} / \mathrm{Dl}$ & $+3,7 \mathrm{mg} / \mathrm{dL}$ & $-6,7 \mathrm{mg} / \mathrm{dL} \mathrm{ns}$ \\
\hline \multicolumn{8}{|l|}{ Metaanálisis } \\
\hline $\begin{array}{l}\text { Chudyc } \\
\text { et al. }{ }^{8}\end{array}$ & N 34 estudios & $\begin{array}{l}\approx 700-800 \text { MET-min-sem } \\
\text { A, R y C vs no ejercicio }\end{array}$ & $\begin{array}{l}\text { A }-0,6 \% \\
\text { R }-0,33 \% \text { ns } \\
C-0,67 \%\end{array}$ & Cambio ns & Cambio ns & Cambio ns & A y $C-27 \mathrm{mg} / \mathrm{dL}$ \\
\hline $\begin{array}{l}\text { Hayashino } \\
\text { et al. }{ }^{9}\end{array}$ & N 42 estudios & $\begin{array}{l}\approx 800 \text { METs-min-sem A, } \\
\text { R y C vs no ejercicio }\end{array}$ & $\begin{array}{l}\text { Global } \\
-0,51 \%\end{array}$ & & $\begin{array}{l}\text { Global }-6,2 \mathrm{mg} / \mathrm{dL} \\
\text { sin diferencias entre } \\
\text { los grupos }\end{array}$ & $\begin{array}{l}\text { Global }+1,54 \mathrm{mg} / \mathrm{dL} \\
\text { sólo significativo en } \\
\mathrm{A}+\mathrm{R}+3,1 \mathrm{mg} / \mathrm{dL}\end{array}$ & $-2,65 \mathrm{mg} / \mathrm{dL} \mathrm{ns}$ \\
\hline $\begin{array}{l}\text { Scott } \\
\text { et al. }{ }^{13}\end{array}$ & N 12 estudios & $A$ vs $R$ & $\begin{array}{l}\text { A }-0,46 \% \\
\text { R }-0,32 \% \\
\text { A-R }-0,14 \%\end{array}$ & $\begin{array}{l}\mathrm{A}-4,2 \mathrm{mg} / \mathrm{dL} \\
\mathrm{R}-8,4 \mathrm{mg} / \mathrm{dL} \\
\mathrm{R}-\mathrm{A}-4,2 \mathrm{mg} / \mathrm{dL} \mathrm{ns}\end{array}$ & $\begin{array}{l}\text { A }-3.09 \mathrm{mg} / \mathrm{dL} \\
\mathrm{R}-1,9 \mathrm{mg} / \mathrm{dL} \\
\text { A-R - } 1,2 \mathrm{mg} / \mathrm{dL} \mathrm{ns}\end{array}$ & $\begin{array}{l}\mathrm{A}+1,16 \mathrm{mg} / \mathrm{dL} \\
\mathrm{R}-0,77 \mathrm{mg} / \mathrm{dL} \\
\mathrm{A}-\mathrm{R}+0,39 \mathrm{~m} / \mathrm{dL} \mathrm{ns}\end{array}$ & $\begin{array}{l}\mathrm{A}-12,4 \mathrm{mg} / \mathrm{dL} \\
\mathrm{R}-29,2 \mathrm{mg} / \mathrm{dL} \\
\mathrm{R}-\mathrm{A}-16,8 \mathrm{mg} / \mathrm{dL} \mathrm{ns}\end{array}$ \\
\hline
\end{tabular}

A: aeróbico; $R$ : resistencia; $C$ : combinados $A+R$; ns: no significativo.

Tabla 1: Efectos del ejercicio en HbA1c y lípidos en pacientes DM2. 
Otros estudios evaluaron los cambios generados en apolipoproteínas y en las características cualitativas de las lipoproteínas. Alam et al. ${ }^{16}$ demostraron que seis meses de ejercicios supervisados en DM2 redujo el pool de Apo B y que ello se debía a una disminución de la velocidad de secreción de VLDL Apo B.

No obstante que el LDL-C en DM2 suele ser normal, existe evidencia de un reducido catabolismo con aumento del "tiempo medio de residencia" (TMR), lo cual incrementa el riesgo aterogénico. El mismo grupo anterior evaluó la cinética de la Apo B unida a LDL y comprobó que, tras seis meses de ejercicio aeróbico supervisado, el colesterol de LDL no cambió, pero hubo un descenso significativo del "tiempo medio de residencia" (TMR) de LDL lo cual correlacionó con un descenso de VLDL y con el aumento del VO2max ${ }^{17}$.

Otros estudios demostraron que el ejercicio aeróbico puede mejorar la eficiencia de HDL en reducir la oxidación de LDL y favorecer la maduración de $\mathrm{HDL}^{18}$.

\section{CONCLUSIONES}

Aún al considerar la heterogeneidad de los estudios puede concluirse que los efectos del ejercicio sobre el perfil lipídico en pacientes DM2 son positivos, aunque modestos, y con un beneficio más manifiesto de los ejercicios aeróbicos y combinados, y sobre todo cuando el volumen y la intensidad del ejercicio es mayor a 800 METsmin-semana (200 min semanales de actividad moderada de 4 a 6 METs de intensidad o 100 min de actividad vigorosa mayor a 8 METs). Además el ejercicio puede generar beneficiosos cambios cualitativos y en la cinética de las lipoproteínas, y cuantitativos de las apoproteínas propios de la dislipidemia diabética.

La presente revisión formará parte del libro "Dislipidemia diabética," Editor: Dr. Yulino Castillo, y ha sido reproducida en esta publicación con la correspondiente previa autorización.

\section{BIBLIOGRAFÍA}

1. Ross R, Blair S, Després JP, Levine B, et al. Importance of assessing cardiorespiratory fitness in clinical practice: a case for fitness as a clinical vital sign. A scientific statement from the American Heart Association. Circulation 2016; 134: e653-e699.

2. Bouchard C, Daw EW, Rice T, Pérusse L, Gagnon J, Province MA, Leon AS, Rao DC, Skinner JS, Wilmore JH. Familial resemblance for VO2max in the sedentary state: the HERITAGE family study. Med Sci Sports Exerc 1998; 30:252-258.
3. Jurca R, Jackson A, Blair S, Haskell W, et al. Assessing cardiorespiratory fitness without performing exercise testing. Am J Prev Med 2005; 29: 185-93.

4. Wei M, Gibbons LW, Kampert JB, Nichaman MZ, Blair SN. Low cardiorespiratory fitness and physical inactivity as predictors of mortality in men with T2DM. Ann Intern Med 2000; 132: 605-611.

5. Piercy KL, Troiano RP, Ballard RM, Carlson SA, Fulton JE, Galuska DA, George SM, Olson RD. The Physical Activity Guidelines for Americans. JAMA 2018.

6. Foster C, Hillsdon M, Thorogood M, Kaur A, Wedatilake T. Interventions for promoting physical activity. Cochrane Database of Systematic Reviews 2005; Issue 1. Art. No.: CD003180. Doi: 10.1002/14651858.CD003180.pub2.

7. Brouwers $B$, Hesselink MKC, Schrauwen P, Schrauweb-Hinderling VB. Effects of exercise training on intrahepatic lipid content in humans. Diabetologia 2016; 59: 2068-2079.

8. Balducci S, Zanuso S, Nicolucci A, De Feo P, Cavallo S, Cardelli P, Fallucca S, Alessi E, Fallucca F, Pugliese G; Italian Diabetes Exercise Study (IDES) Investigators. Effect of an intensive exercise intervention strategy on modifiable cardiovascular risk factors in subjects with type 2 diabetes mellitus: a randomized controlled trial: the Italian Diabetes and Exercise Study (IDES). Arch Intern Med 2010; 170:1794-803.

9. Chudyk A, Petrella R. Effects of exercise on cardiovascular risk factors in type 2 diabetes. A meta-analysis. Diabetes Care 2011; 34:1228-1237.

10. HayashinoY, Jackson JL, Fukumori N, Nakamura F, Fukuhara S. Effects of supervised exercise on lipid profiles and blood pressure control in people with type 2 diabetes mellitus: A metaanalysis of randomized controlled trials. Diabetes Research and Clinical Practice 2012; 98:349-60

11. Sigal RJ, Armstrong MJ, Bacon SL, Boulé NG, Dasgupta K, Kenny GP, Riddell MC; Diabetes Canada Clinical Practice Guidelines Expert Committee. Physical activity and diabetes. Can J Diabetes 2018 Apr; 42 Suppl 1:S54-S63.

12. Srikanthan $P$, Karlamangla $A$. Muscle mass index as a predictor of longevity in older adults. Am J Med 2014; 127: 547-53.

13. Scott D, de Courten B, Ebeling P. Sarcopenia: a potential cause and consequence of type 2 diabetes in Australia's ageing population? Med J Aust 2016; 205: 329-333.

14. Yang Z, Scott CA, Mao C, Tang J, Farmer AJ. Resistance exercise vs aerobic exercise for type 2 diabetes: a systematic review and meta-analysis. Sports Med 2014; 44:487-499.

15. Schwingshackl L, Missbach B, Dias S, König J, Hoffmann G. Impact of different training modalities on glycaemic control and blood lipids in patients with type 2 diabetes: a systematic review and network meta-analysis. Diabetologia 2014 Sep;57(9):1789-97.

16. Alam S, Stolinski M, Pentecost C, Boroujerdi MA, Jones RH, Sonksen $\mathrm{PH}$, Umpleby AM. The effect of a six-month exercise program on very low-density lipoprotein apolipoprotein B secretion in type 2 diabetes. J Clin Endocrinol Metab 2004; 89:688-694.

17. Stolinski M, Alam S, Jackson NC, Shojaee-Moradie F, Pentecost C, Jefferson W, Christ ER, Jones RH, Umpleby M. Effect of 6-month supervised exercise on low-density lipoprotein apolipoprotein B kinetics in patients with type 2 diabetes mellitus. Metabolism Clinical and Experimental 2008; 57:1608-1614.

18. Ribeiro I, Iborra RT, Neves M, Lottenberg A, Charf AM, Nunes VS, Negra CE, Nakandakare ER, Quinta EC, Passarelli M. HDL atheroprotection by aerobic exercise training in type 2 diabetes mellitus. Med Sci Sports Exerc 2008; 40:779-786. 


\section{Fe de erratas}

En la Revista de la Sociedad Argentina de Diabetes, Vol. 51, № 2, Mayo-agosto de 2017, pág. 41, donde se encuentra la Figura 2, por un error involuntario se invirtieron los colores de las referencias de: "Total", "No hiperglucémicos" e "Hiperglucémicos." La Figura 2 correcta es la siguiente:

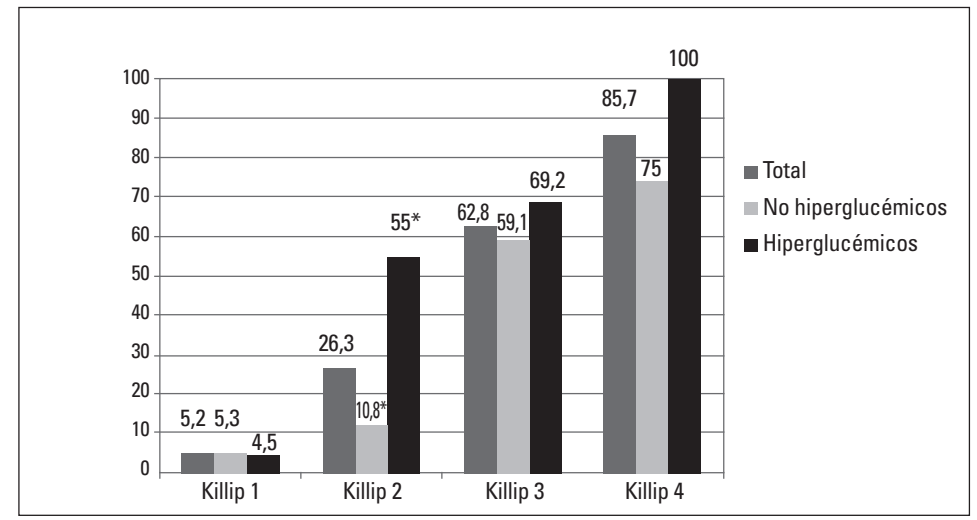

${ }^{*} p<0,01$.

Figura 2: Muestra la evaluación de mortalidad total, en pacientes no hiperglucémicos y en pacientes hiperglucémicos, por cada punto de la escala de Killip y Kimball. 